\title{
High-brightness all-fiber Raman lasers directly pumped by multimode laser diodes
}

\author{
S. A. Babin ${ }^{1,2}$ \\ ${ }^{1}$ Institute of Automation and Electrometry, SB RAS, Novosibirsk 630090, Russia \\ ${ }^{2}$ Novosibirsk State University, Novosibirsk 630090, Russia \\ (Received 8 November 2018; revised 15 December 2018; accepted 20 December 2018)
}

\begin{abstract}
High-brightness fiber laser sources usually utilize active rare-earth-doped fibers cladding-pumped by multimode laser diodes (LDs), but they operate in limited wavelength ranges. Singlemode-passive-fiber based Raman lasers are able to operate at almost any wavelength being pumped by high-power fiber lasers. One of the interesting possibilities is to directly pump graded-index (GRIN) multimode passive fibers by available high-power multimode LDs at 915-940 nm, thus achieving high-power Raman lasing in the wavelength range of 950-1000 nm, which is problematic for rare-earthdoped fiber lasers. Here we review the latest results on the development of all-fiber high-brightness LD-pumped sources based on GRIN fiber with in-fiber Bragg gratings (FBGs). The mode-selection properties of FBGs inscribed by fs pulses supported by the Raman clean-up effect result in efficient conversion of multimode pump into a high-quality output beam at 9xx nm. GRIN fibers with core diameters $62.5,85$ and $100 \mu \mathrm{m}$ are compared. Further scaling capabilities and potential applications of such sources are discussed.
\end{abstract}

Keywords: beam cleaning; fiber laser; high brightness; laser diode pumping; Raman laser

\section{Introduction}

High-brightness fiber laser sources based on active rareearth (RE)-doped fibers are characterized by excellent output characteristics and unique performance enabling their wide use for scientific and industrial applications ${ }^{[1]}$. However, they operate at specific wavelengths defined by the dopant gain bandwidth that limits their application range. Singlemode-passive-fiber based Raman lasers are able to operate at almost any wavelength defined by the pump wavelength, Stokes shift and number of cascades at Raman conversion $^{[2]}$. Raman fiber lasers (RFLs) are usually pumped into the singlemode-passive-fiber core by RE-doped fiber lasers operating in singlemode regime with fundamental transverse mode output. Using for pumping the most powerful Yb-doped fiber laser (YDFL) operating around $1 \mu \mathrm{m}$, it is possible to obtain Raman lasing almost in the whole transmission window of silica fibers $(1-2 \mu \mathrm{m})$, thanks to the efficient cascaded generation of higher-order Stokes components. In their turn, high-power RE-doped fiber lasers are usually cladding-pumped by multimode (MM) laser diodes (LDs). Such a complex pumping scheme leads to a rather complicated design of RFLs. An interesting possibility

Correspondence to: S. A. Babin, Institute of Automation and Electrometry, SB RAS, Novosibirsk 630090, Russia. Email: babin@iae.nsk.su to simplify the RFL design is its direct pumping by cheap and reliable high-power MM LDs, similar to the LD-pumped RE-doped fiber lasers ${ }^{[1]}$. Though it is possible to employ cladding pumping, this technique requires the development of special double-clad passive fibers. In this way, Raman lasing at $1120 \mathrm{~nm}$ with output power of $100 \mathrm{~W}$ and beamquality parameter $M^{2} \sim 1.6$ was demonstrated with an MM YDFL as a pump source ${ }^{[3]}$, whereas at direct diode pumping only $6 \mathrm{~W}$ output power was obtained by the same group ${ }^{[4]}$.

From technical and economical points of view, it is attractive to use standard graded-index (GRIN) MM fibers which are widely used in telecom. In this case, one can directly couple the MM radiation of high-power LDs into the MM GRIN-fiber core. In addition, using commercially available MM LDs with operating wavelengths of 915$940 \mathrm{~nm}$, it is possible to obtain high-power Raman lasing in the wavelength range of 950-1000 nm, which is problematic for RE-doped fiber lasers. At the same time, output beam quality of the MM RFL may be high enough due to the beam clean-up effect at Raman conversion of continuous wave $(\mathrm{CW})$ radiation in GRIN fibers ${ }^{[5,6]}$. Since the power of commercial 9xx-nm LDs exceeds $100 \mathrm{~W}$ level for a single unit pigtailed by a $105-\mu \mathrm{m}$ core fiber, the MM RFL with direct LD pumping became feasible. 
The concept of LD-pumped GRIN-fiber based RFLs has been developed in two different directions. First direction continues the study initiated in Ref. [5] with a focus on the opportunities of high-quality beam generation using fiber Bragg gratings (FBGs) as cavity mirrors ${ }^{[7-10]}$, whereas second one aims at high-power capabilities based on freespace pump coupling and bulk mirrors cavity ${ }^{[4,11,12]}$. In the last case, up to $154 \mathrm{~W}$ power is obtained at $\sim 1020 \mathrm{~nm}^{[12]}$, but Raman output at this wavelength is not as interesting, because higher power and narrower linewidth are available from YDFLs ${ }^{[13,14]}$. In addition, in spite of significant improvement of the output beam quality $\left(M^{2}=4-8\right)$ in comparison with that for the pump beam $\left(M^{2} \sim 20\right)$ due to the Raman clean-up effect in GRIN fibers, it is far from singlemode regime.

The first experiments with a cavity FBG UV-inscribed in telecom GRIN fiber of $62.5-\mu \mathrm{m}$ core pumped by a $940-\mathrm{nm}$ MM LD resulted in $\sim 3 \mathrm{~W} 980-\mathrm{nm}$ output of much better beam quality generated with Fresnel reflection at the output end $^{[7]}$. Using angled cleaving of the output fiber end, random lasing in half-open cavity of such LD-pumped GRIN-fiber lasers has been also demonstrated ${ }^{[8]}$. As a next step, a 915$\mathrm{nm}$ LD pumping of a $62.5-\mu \mathrm{m}$ GRIN fiber with two UVinscribed FBGs (highly reflective and output one) enabled generation of 954-nm Stokes radiation ${ }^{[9]}$. Implementation of a special output FBG inscribed by a point-by-point femtosecond (fs) technique in the central part $(1 \times 10 \mu \mathrm{m})$ of the 62.5- $\mu \mathrm{m}$ GRIN-fiber core resulted in Raman lasing with output power of $10 \mathrm{~W}$ at $954 \mathrm{~nm}$ in nearly singlemode $\left(M^{2} \sim 1.2\right)^{[10]}$, due to the mode-selective properties of such FBG.

Here we review our recent results on the development of simple and reliable all-fiber configuration of such RFL having high beam quality and high power simultaneously, due to the fiber coupling of several LDs enabling highpower pumping of a GRIN fiber and FBGs with special 3D structure featured by mode-selective properties. GRIN fibers with core diameters $62.5,85$ and $100 \mu \mathrm{m}$ are compared. Further scaling capabilities and potential applications of such sources are discussed.

\section{Experimental scheme and operating principles}

Experimental scheme of the studied LD-pumped MM GRIN-fiber RFL is shown in Figure 1. Instead of a bulkoptics scheme (shown in the dashed box) for coupling of LD pump radiation to the GRIN-fiber core used in the first experiments $^{[7-10]}$, we implemented a fiber coupling scheme based on a fusion spliced combiner for 3 LDs with GRINfiber output port of $100 \mu \mathrm{m}$ core diameter ${ }^{[15]}$. Coupling of several diodes (LD1-LD3) through the MM fiber pump combiner directly to the Raman GRIN fiber allows one to increase the pump power. In addition, using the Raman

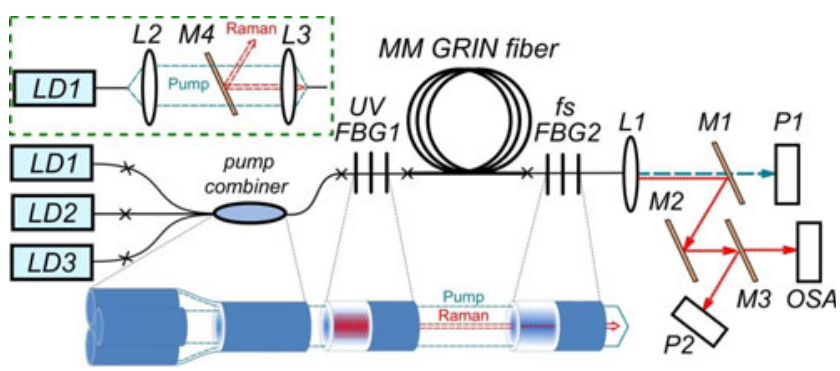

Figure 1. All-fiber scheme of MM GRIN-fiber Raman laser with cavity formed by HR UV-FBG1 and output fs-FBG2 with LD pumping through the fiber pump combiner (or bulk-optic pump coupling scheme ${ }^{[10]}$ shown in the inset).

GRIN fiber of larger-core diameter results in sufficient reduction of pump coupling losses. In-fiber gratings UVFBG1 and fs-FBG2 are inscribed in the studied Raman GRIN fiber by ultraviolet- and femtosecond-FBG fabrication technologies, respectively; see Refs. [10, 15] for details. The obtained all-fiber configuration has obvious advantages such as compactness, ease of operation, low cost, reliability, long-term stability in combination with flexibility of the output fiber end. Output beam consisted of the Stokes and transmitted pump radiation split by dichroic mirrors M1-M3 is analyzed by means of power meters (P1, P2) and optical spectrum analyzer replaced by Thorlabs $M^{2}$-meter for beamquality measurements.

A comparison of the $1.1 \mathrm{~km} 62.5-\mu \mathrm{m}$ GRIN RFL with pump coupling via fiber spliced combiner ${ }^{[15]}$ and that one with bulk optics ${ }^{[10]}$ has shown that the generation threshold increases from $\sim 40 \mathrm{~W}$ (bulk optics) to $\sim 50 \mathrm{~W}$ (allfiber). At the same time, the RFL efficiency and maximum Stokes power at $954 \mathrm{~nm}$ also increase (40 to $47 \%$ and 10 to $18 \mathrm{~W}$, respectively) after the implementation of fiber coupling which eliminates reflection from the input fiber facet thus increasing the threshold for the 2nd Stokes wave.

Then we tested the developed all-fiber MM RFL scheme with the use of GRIN Raman fiber of different core diameters: $62.5,85^{[15]}$ and $100^{[16]} \mu \mathrm{m}$, in which corresponding highly reflective (HR) UV-FBG1 and output fs-FBG2 have been fabricated. The point-by-point fs inscription technology based on nonlinear (multi-photon) mechanism of refractive index change enables modification of a small area near the center of GRIN-fiber core, and therefore an output coupler made by this technology preferably reflects the fundamental transverse mode. At the same time, HR FBG made by this technology has high losses, which is why we used UV-inscribed HR FBGs with relatively low losses. The measured FBG spectra in different GRIN fibers are compared in Figure 2. One can see that for all fs-FBGs the maximum reflection is provided for the longer-wavelength peak corresponding to the fundamental transverse mode. The periodic short-wavelength resonances correspond to the FBG coupling with different groups of higher-order modes. The spectral separation between the groups decreases 

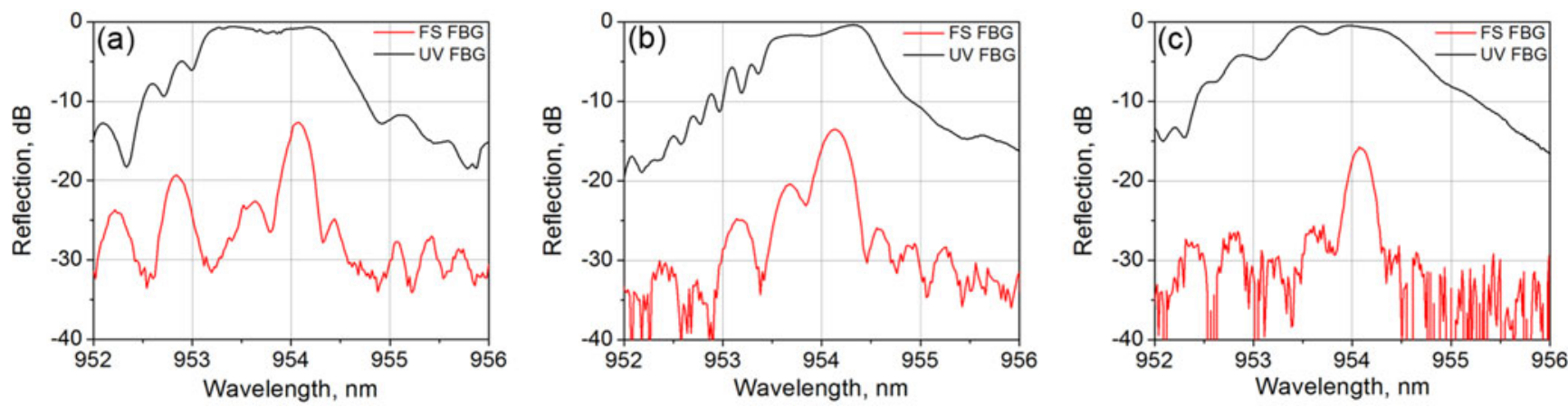

Figure 2. Comparison of HR UV-FBG and output fs-FBG inscribed in GRIN fibers of different core diameters: (a) $62.5 \mu \mathrm{m}^{[10]}$, (b) $85 \mu \mathrm{m}^{[15]}$ and (c) $100 \mu \mathrm{m}^{[16]}$.
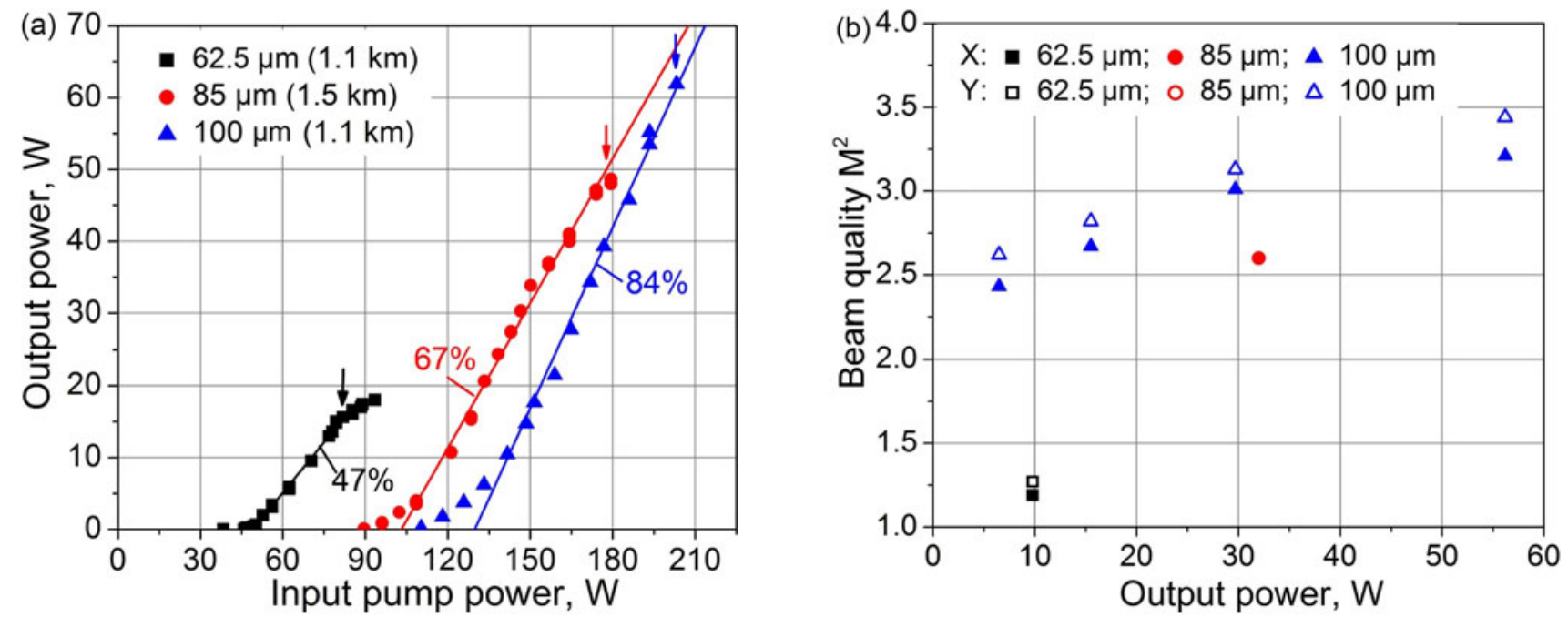

Figure 3. (a) Output Stokes power at $954 \mathrm{~nm}$ (filled symbols) as a function of input (coupled to GRIN fiber) LD pump power and (b) corresponding beamquality parameter $M^{2}$ as a function of Stokes power for different GRIN-fiber core diameters. Arrows mark the threshold of the 2nd-order Stokes wave generation at $996 \mathrm{~nm}$.

with increasing core diameter (amounting to about $0.6,0.5$ and $0.4 \mathrm{~nm}$ for $62.5,85$ and $100 \mu \mathrm{m}$ core, respectively) in correspondence with the well-known formula ${ }^{[17]}$

$$
\Delta \lambda=\lambda^{2} \cdot N A /\left(\pi d n_{1}^{2}\right),
$$

where $\lambda$ is the wavelength of fundamental mode, $N A$ is numerical aperture, $d$ is the core diameter, and $n_{1}$ is the refractive index of cladding. It should be also noted that HR UV-FBGs have rather smooth profile with strongly overlapping mode resonances, whereas output fs-FBG has clear resonant structure with sufficient discrimination of higher-order modes (about $-10 \mathrm{~dB}$ for all core diameters) relative to the fundamental mode peak. These features of cavity FBGs enable efficient fundamental mode selection in MM GRIN-fiber RFLs at relatively low transmission of generated Stokes power toward the pump LDs.

Power, spectral and beam-quality characteristics of the generated Stokes radiation at $954 \mathrm{~nm}$ have been measured and compared for different Raman GRIN fibers keeping the same three 915-nm pump LDs and the same pump combiner (of $100 \mu \mathrm{m}$ core). Note that the pump coupling losses are reduced with the increasing core diameter of the Raman GRIN fiber amounting to about $75 \%, 50 \%$ and $25 \%$ for $62.5,85$ and $100 \mu \mathrm{m}$ core, respectively, thus increasing the available coupled pump power.

\section{Raman lasing in GRIN fibers of different core diameters}

The output power at $954 \mathrm{~nm}$ as a function of input (coupled to the Raman fiber) pump power in different GRIN fibers of optimal length is shown in Figure 3 together with the measured beam-quality parameter $M^{2}$ for the generated Stokes beam.

Optimal Raman fiber length is defined by the 2nd Stokes threshold and the available pump power from the same three 915-nm LDs given that the losses at pump coupling to the Raman fiber decrease with its increasing core diameter. So, the maximum input pump power is about 90,180 and $240 \mathrm{~W}$ for $62.5,85$ and $100 \mu \mathrm{m}$ GRIN fibers, respectively. As the 2nd Stokes threshold can be shifted to higher powers by 

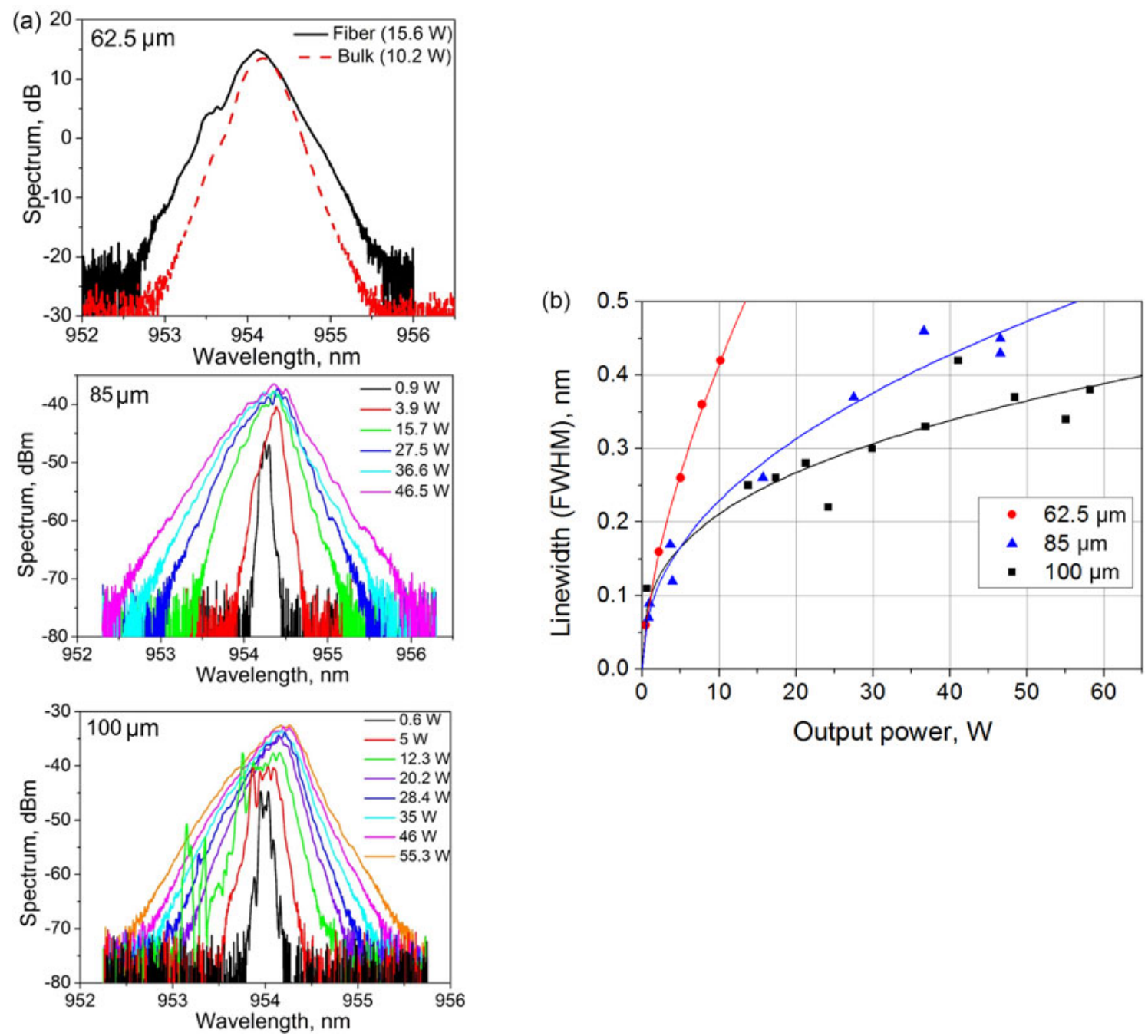

Figure 4. (a) Output spectra for GRIN-fiber Raman laser with $62.5,85$ and $100 \mu \mathrm{m}$ cores at different Stokes powers. (b) Corresponding $-3 \mathrm{~dB}$ linewidth as a function of the generated Stokes power at $954 \mathrm{~nm}$ fitted by curves $\sim x^{a}$ with best-fit parameter $a=0.64(62.5 \mu \mathrm{m}) ; 0.45(85 \mu \mathrm{m}) ; 0.34(100 \mu \mathrm{m})$.

shortening GRIN fiber, we made it so that the 2nd Stokes threshold (marked by arrow in Figure 3(a)) becomes closer to the maximum input pump power. This gives the value of optimal length appearing to be nearly the same for all core diameters of GRIN fiber. At the optimal length, the output Stokes powers (and slope efficiency for conversion of input pump to Stokes output power) take their maximum values at the available pump power, amounting to about 18 , 49 and $62 \mathrm{~W}$ (and 47\%, 67\% and 84\%), respectively; see Figure 3(a). Herewith, the measured beam-quality parameter $M^{2}$ slightly worsens with increasing core diameter amounting to about 1.3, 2.5 and 3 for the tested GRIN fibers; see Figure 3(b). Detailed measurements of beam quality for 100$\mu \mathrm{m}$ GRIN fiber by fitting of the focused beam waist shape show that it is nearly the same for $x$ and $y$ beam axes (solid and empty symbols) and is only slightly varying in the range $M^{2}=2.5-3.2$ with increasing output power from $\sim 10$ to $\sim 60 \mathrm{~W}$. At that, the measured quality parameter for the pump radiation at maximum power amounts to $M^{2} \sim 32$ (the quality worsens after pump combiner). It means that the brightness defined as

$$
B=P /\left(M^{2} \lambda\right)^{2}
$$

is enhanced for the Raman output beam as compared to the input pump beam by $\sim 20$ times due to the joint action of Raman beam clean-up effect and mode-selective properties of cavity FBGs. The enhancement factor related to the coupled pump power is nearly the same for all GRIN fibers, but with an account for the pump coupling efficiency the largercore GRIN fibers are more suitable for the development of high-brightness high-power sources at $9 \mathrm{xx} \mathrm{nm}$. Note that the transmitted LD pump power at the maximum generated Stokes power is nearly the same $(30-40 \mathrm{~W})$ for all fibers that also makes larger-core GRIN fibers more preferable for LD brightness enhancement.

The measured Stokes spectrum for all GRIN fibers has a shape close to the hyperbolic secant with exponential wings (see Figure 4(a)), just like the spectrum of conventional 

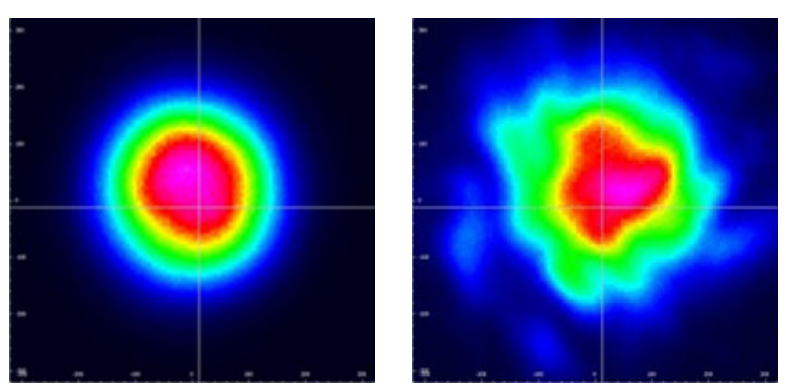

(a)

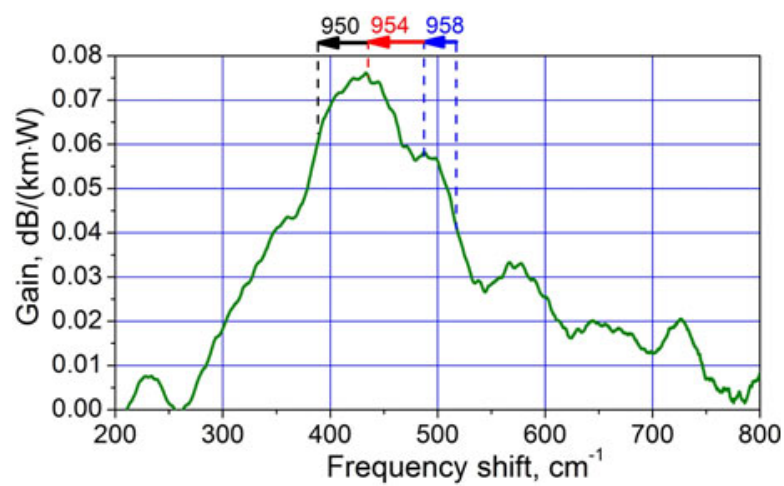

Figure 5. (a) Stable and unstable beam profiles at low power $(13 \mathrm{~W})$ for operation at $950 \mathrm{~nm}$ (left) and $958 \mathrm{~nm}$ (right) in 100- $\mu \mathrm{m}$ GRIN RFL, respectively. (b) Measured Raman gain profile with corresponding marks illustrating 100- $\mu \mathrm{m}$ GRIN RFL operation with 950-, 954- and 958-nm FBGs ${ }^{[16]}$

Raman lasers based on singlemode fibers ${ }^{[18]}$. The only sign of higher-order transverse mode impurities consists in visible asymmetry of the spectral profile that is more pronounced for 85- and 100- $\mu \mathrm{m}$ GRIN fibers in correspondence with the beam-quality data (Figure 3(b)). The width of the generated spectrum is defined by the reflection spectrum of cavity FBG (Figure 2) and nonlinear-optical effects resulting in its power broadening. The extracted $-3 \mathrm{~dB}$ linewidth grows with increasing power nonlinearly reaching nearly the same value for three core sizes, but at sufficiently different Stokes powers. Taking that the power broadening is defined by the effect of self-phase modulation (SPM) that is proportional to the laser beam intensity, this means that the effective beam area is sufficiently larger in larger-core GRIN fibers. An additional effect reducing the RFL linewidth is the group velocity dispersion (GVD) in relation to the nonlinear effects (SPM, Raman gain). If its relative contribution increases, the power low changes from linear to nonlinear one with a cubic root law in the limiting case of strong dispersion (see Ref. [19] and citation therein). In case of GRIN fibers with different cores, the degree of the root law $x^{a}$ amounts to $a=0.64,0.45$ and 0.34 for $62.5,85$ and $100-\mu \mathrm{m}$ fibers, respectively, thus confirming increasing role of GVD in larger-core GRIN fibers.

It should be also noted that the $-3 \mathrm{~dB}$ linewidth remains to be smaller than the corresponding distance between the neighboring groups of transverse modes of GRIN fiber.
Besides, in case of $100-\mu \mathrm{m}$ GRIN fiber the laser spectrum becomes unstable at low powers, but stabilizes at high powers (quite opposite to the mode instability effect in RE-doped fiber lasers ${ }^{[20]}$ ). At that, both the spectral (Figure 4(a), lower picture) and spatial (Figure 5(a)) profiles exhibit the signs of high-order transverse mode appearance time to time, while at high powers the profiles are stable consisting of constant group of low-order modes with the main contribution from the fundamental modes, just like for smaller-core GRIN fibers in a broad range of powers.

To clarify the mechanism of the low-power mode instability in 100- $\mu \mathrm{m}$ GRIN fiber, Raman generation with FBGs centered at $950 \mathrm{~nm}$ and $958 \mathrm{~nm}$ (slightly shifted from the Raman gain maximum to the short and to the long wavelengths, respectively) was tested in Ref. [16]. It has been found that the unstable behavior with random appearance of high-order transverse modes is observed in different power domains for different generation lines. The spectrum stabilizes at $>35 \mathrm{~W}$ for $958 \mathrm{~nm}$, at $>18 \mathrm{~W}$ for $954 \mathrm{~nm}$ and at $>8 \mathrm{~W}$ for $950 \mathrm{~nm}$, thus demonstrating that the transverse mode competition depends on the FBG wavelength position within the Raman gain spectral profile (Figure 5(b)). The weakest instability effect is observed at the short-wavelength slope of Raman gain at 950-nm operation when the gain coefficient for higher-order modes is lower than that for the fundamental one. Note that the Raman shift between LD and FBG wavelengths slightly decreases with increasing LD power which experience "red" wavelength shift at high powers.

\section{Random Raman lasing in GRIN fibers}

As shown in the previous section, 2nd-order Stokes wave generation threshold due to the random distributed feedback via Rayleigh backscattering along the GRIN fiber (and probably some reflection from splice points, etc.) limits the maximum power of the 1st Stokes Raman lasing. This limitation may be moved to higher powers by shortening the fiber accompanied by corresponding increase of the coupled pump power and/or by inserting additional losses for the 2 nd Stokes wave, similar to singlemode fibers ${ }^{[21]}$.

However, cascaded generation of the 2nd Stokes order may be treated not only as a limiting effect, but also as an opportunity to obtain new laser wavelengths, similarly to cascaded random lasing in singlemode fibers ${ }^{[19]}$. In GRIN fibers this regime is also interesting from the point of further beam-quality improvement. Such experiments have been performed by using an additional input HR FBG at $996 \mathrm{~nm}$ inserted in the scheme of Figure 1, thus forming a half-open cavity with random distributed feedback for the 2nd Stokes wave. At that, the 1 st Stokes wave generated in normal FBG cavity at $954 \mathrm{~nm}$ serves as a pump for that. The obtained 2nd Stokes output power at $996 \mathrm{~nm}$ is shown in Figure 6(a) 

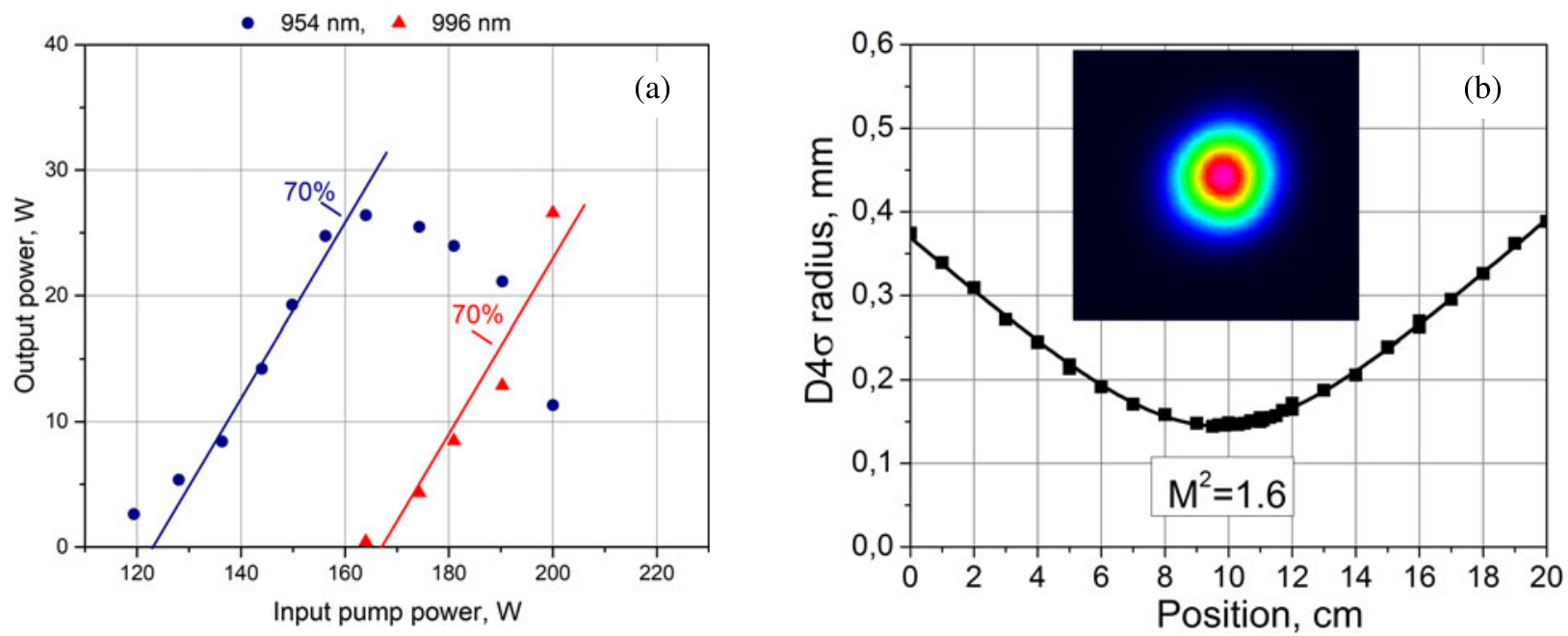

Figure 6. (a) Output power and (b) beam-quality characteristics of 2nd-order random Raman lasing at $996 \mathrm{~nm}$ in $100-\mu \mathrm{m}$ GRIN RFL together with power of the 1 st Stokes output at $954 \mathrm{~nm}$.

together with the 1 st Stokes power as a function of input LD pump power. After the 2nd threshold pump power of $\sim 160 \mathrm{~W}$, the measured output power at $996 \mathrm{~nm}$ selected by additional dichroic mirror grows rapidly with an average slope of about $70 \%$ that is nearly the same as that for the 1 st Stokes wave (before the 2nd Stokes threshold). Maximum power at $996 \mathrm{~nm}$ amounts to $27 \mathrm{~W}$. At the same time, the beam quality for random lasing at $996 \mathrm{~nm}$ appears to be sufficiently better than that at $954 \mathrm{~nm}$ amounting to about $M^{2} \sim 1.6$ without significant changes in all power range. So, random distributed feedback additionally improves the beam quality, as it was also mentioned at low-power 1st Stokes random lasing ${ }^{[8]}$. This effect may be explained by stronger Rayleigh-backscattering feedback for the fundamental transverse mode, improving its selection in addition to the effects of Raman beam clean-up and mode-selective FBGs. It appears also possible to obtain random lasing at significantly shifted wavelength $(978 \mathrm{~nm})$ with relatively good parameters, which are measured to be much better than the normal 2nd-order Raman lasing in the linear cavity formed by two FBGs; for more details of this study, see Ref. [22]. The obtained high beam quality made it possible to realize efficient frequency doubling in PPLN nonlinear crystal, thus generating blue laser light near $489 \mathrm{~nm}$; the details of this study will be published elsewhere.

\section{Conclusion}

Thus, we have demonstrated possibilities to develop highbrightness 9xx-nm fiber sources based on the passive MM GRIN fiber directly pumped by high-power MM LDs. Allfiber configuration of such diode-pumped RFL is shown to be possible with the use of FBGs inscribed in the MM GRIN-fiber core and LD coupling via spliced fiber pump combiner. It has been demonstrated that the mode-selection properties of FBGs inscribed by fs pulses in the central part of fiber core (supported by the Raman clean-up effect) result in efficient conversion of MM LD pump radiation coupled to the fiber $\left(M^{2} \sim 30\right)$ into a high-quality output beam at $9 \times x \mathrm{~nm}$ with brightness enhancement factor of about 20. GRIN fibers with core diameters $62.5,85$ and $100 \mu \mathrm{m}$ have been compared. With the core enlargement, the pump to Stokes conversion efficiency increases from $47 \%$ to $84 \%$ at the expense of slight enlargement of beamquality parameter $M^{2}$ (from 1.3 to $\sim 3$ ). At that, maximum output power at $954 \mathrm{~nm}$ amounts to 18,49 and $62 \mathrm{~W}$ for 62.5, 85 and $100 \mu \mathrm{m}$ GRIN fiber, respectively, pumped by the same $915-\mathrm{nm}$ LDs. The maximum power is defined by the losses at fiber coupling of LDs to corresponding GRIN fiber being lower for larger-core fibers. The laser linewidth appears to be nearly the same $(\sim 0.4 \mathrm{~nm})$ for all GRIN fibers at corresponding maximum output power that is reasoned by increasing effective beam area in larger-core fibers. It has been also observed that the beam quality and spectral width are only weakly varying in the broad range of powers (10-60 $\mathrm{W}$ for $100-\mu \mathrm{m}$ fiber), thus indicating the formation of stable group of coupled low-order modes. At that, the power broadening law becomes close to cubic root with increasing core diameter (power degree decreases from 0.64 to 0.34 ), thus indicating increasing role of GVD in largercore GRIN fibers. Experiments on 2nd-order random lasing at 996 and $978 \mathrm{~nm}$ and second harmonic generation have been also endeavored, which demonstrate a possibility to generate new laser wavelengths with improved beam quality in the developed RFL scheme.

Note that these are only the first steps demonstrating basic principles of the new type of $\mathrm{CW}$ high-power fiber laser. The next steps toward a better pump coupling at higher powers and a better fundamental mode selection will 
result in further improvements in optical efficiency, output power and brightness of such sources. This makes it very attractive for applications, such as efficient/bright source for pumping solid-state/fiber lasers, second harmonic generation with generation of visible light and corresponding application for laser displays and bio-medical imaging. Interesting fundamental challenges also appear, e.g., investigation of mode instability in passive GRIN fibers, which may behave differently from that in step-index active fibers, thus breaking the existing limits of high-power fiber lasers.

\section{Acknowledgements}

The author acknowledges financial support of Russian Science Foundation (grant 14-22-00118) and valuable assistance of S. I. Kablukov, E. A. Evmenova, A. G. Kuznetsov, A. Wolf and S. R. Abdullina during the preparation of this paper.

\section{References}

1. D. J. Richardson, J. Nilsson, and W. A. Clarkson, J. Opt. Soc. Am. B 27, B63 (2010).

2. E. M. Dianov and A. M. Prokhorov, J. Sel. Top. Quantum Electron. 6, 1022 (2000).

3. C. A. Codemard, J. Ji, J. K. Sahu, and J. Nilsson, Proc. SPIE 7508, 75801N (2010).

4. T. Yao, A. V. Harish, J. K. Sahu, and J. Nilsson, Appl. Sci. 5, 1323 (2015).

5. S. H. Baek and W. Roh, Opt. Lett. 29, 153 (2004).

6. N. B. Terry, T. G. Alley, and T. H. Russell, Opt. Express 15, 17509 (2007).
7. S. I. Kablukov, E. I. Dontsova, E. A. Zlobina, I. N. Nemov, A. A. Vlasov, and S. A. Babin, Laser Phys. Lett. 10, 085103 (2013).

8. S. A. Babin, E. I. Dontsova, and S. I. Kablukov, Opt. Lett. 38, $3301(2013)$.

9. E. A. Zlobina, S. I. Kablukov, M. I. Skvortsov, I. N. Nemov, and S. A. Babin, Laser Phys. Lett. 13, 035102 (2016).

10. E. A. Zlobina, S. I. Kablukov, A. A. Wolf, A. V. Dostovalov, and S. A. Babin, Opt. Lett. 42, 9 (2017).

11. Y. Glick, V. Fromzel, J. Zhang, A. Dahan, N. Ter-Gabrielyan, R. K. Pattnaik, and M. Dubinskii, Laser Phys. Lett. 13, 065101 (2016).

12. Y. Glick, V. Fromzel, J. Zhang, N. Ter-Gabrielyan, and M. Dubinskii, Appl. Opt. 56, B97 (2017).

13. Y. Glick, Y. Sintov, R. Zuitlin, S. Pearl, Y. Shamir, R. Feldman, Z. Horvitz, and N. Shafir, J. Opt. Soc. Am. B 33, 1392 (2016).

14. M. Jiang, P. Zhou, H. Xiao, and P. Ma, High Power Laser Sci. Eng. 3, e25 (2015).

15. E. A. Zlobina, S. I. Kablukov, A. A. Wolf, I. N. Nemov, A. V. Dostovalov, V. A. Tyrtyshnyy, D. V. Myasnikov, and S. A. Babin, Opt. Express 25, 12581 (2017).

16. E. A. Evmenova, S. I. Kablukov, I. N. Nemov, A. A. Wolf, A. V. Dostovalov, V. A. Tyrtyshnyy, D. V. Myasnikov, and S. A. Babin, Laser Phys. Lett. 15, 095101 (2018).

17. T. Mizunami, T. V. Djambova, T. Niiho, and S. Gupta, J. Lightwave Technol. 18, 230 (2000).

18. S. A. Babin, D. V. Churkin, A. E. Ismagulov, S. I. Kablukov, and E. V. Podivilov, J. Opt. Soc. Am. B 24, 1729 (2007).

19. S. A. Babin, E. A. Zlobina, S. I. Kablukov, and E. V. Podivilov, Sci. Rep. 6, 22625 (2016).

20. C. Jauregui, J. Limpert, and A. Tünnermann, Nat. Photon. 7, 861 (2013).

21. J. Song, H. Xu, J. Ye, H. Wu, H. Zhang, J. Xu, and P. Zhou, Sci. Rep. 8, 10942 (2018).

22. E. A. Evmenova, A. G. Kuznetsov, I. N. Nemov, A. A. Wolf, A. V. Dostovalov, S. I. Kablukov, and S. A. Babin, Sci. Rep. 8, 17495 (2018). 